\title{
Theoretical, unified derivation of both the integer quantum Hall effect and fractional quantum Hall effect
}

\author{
Shinichi Ishiguri, Ph.D. \\ Nihon University \\ 1-2-1 Izumi-Cho, Narashinoshi, Chiba 275-8575 JAPAN \\ Email: shinichi.ishiguri@gmail.com
}

\begin{abstract}
In this paper, using the two integers that describe the stationary 2-dimensional wave and the charge quantization along with the balance between the Lorentz force and electrical force, we succeed in deriving the fractional quantum Hall effect and the integer quantum Hall effect; we find that the latter exists as a special case of the former. Moreover, using the derived expression describing the fractional quantum Hall effect, a relationship between the plateau in the resistivity of the sample and the applied magnetic field is obtained. The findings of this model agree well with experimental measurements. Because the two integers that describe the stationary 2-dimensional wave and the charge quantization along with the force balance have concrete physical meanings in this work, we could provide a clear picture of the origin of both the integer quantum Hall effect and the fractional quantum Hall effect.
\end{abstract}

Keywords: Integer quantum Hall effect, Fractional quantum Hall effect, stationary wave, nodes in stationary wave, quantization of electric charge, the plateau of quantized resistivity

\section{Introduction}

K. von Klitzing discovered the integer quantum Hall effect [1], which is a phenomenon that is observed in 2-dimensional semiconductor samples. This effect is related to the quantization of the Hall resistance with extremely high stability. This phenomenon occurs under a strong applied magnetic field at cold temperatures.

By improving the mobility and applying larger magnetic fields, the fractional quantum Hall effect [2] was found. The one-third quantization of the Hall resistance was first found and later, other various fractional numbers quantization were also found. The origin of the integer quantum Hall effect was described in detail by Laughlin and Buttiher [3].

The explanation of the fractional quantum Hall effect was obtained by considering the Laughlin wave function (i.e., quantum liquids), Chern-Simons Ginzburg-Landau (GL) theory, and fractional statistics [4-7]. The Laughlin wave function was based on the assumptions that there are many 
Coulomb force interactions between electrons and that electrons were constrained locally. However, the Chern-Simons theory assumes that an electron accompanies a magnetic flux. This implies that, in this theory, multipole fermions were handled. The mean field theory was then applied to these fermions. The fractional statistics assumes a novel particle called an anyon, which behaves in a manner distinct from both Fermi and Bose statistics.

It was later investigated whether the fractional quantum Hall effect was related to Cooper pairs and superconductivity [8-9].

The issues with the above methods are:

1. The method of giving fractional numbers is complex and unnatural. According to the above theories, to obtain fractional numbers, the concept of "hierarchy" must be introduced, which cannot produce the fractional numbers successively.

2. Moreover, in Ref. [10], the plateaus of the quantization vs. applied magnetic fields were measured, but the above-mentioned theories are unable to explain or predict this measurement. This failing is crucial and thus a further theory has been expected.

Motivated by the above items 1 and 2, this paper describes the fractional quantum Hall effect and explains that the integer quantum Hall effect is a special case of the fractional quantum Hall effect. Moreover, the fractional numbers are naturally provided without invoking the notion of hierarchy. Furthermore, the plateaus observed in the measurements of the quantization vs. applied magnetic fields are accurately predicted. The aim of the present paper is to provide concrete theoretical basis that explains both the integer quantum Hall effect and the fractional quantum Hall effect.

\section{Theory}

In Fig. 1, the model system considered here is shown. A magnetic field $B_{z}$ is applied and current density is supplied by an electric field $\mathrm{E}_{\mathrm{y}}$. In Fig. 1, according to the motion of an electron, there are two forces; electrical force, $F_{E}$, and Lorentz force, $F_{B}$. "Force balance" simply implies that $F_{E}=F_{B}$. Moreover, in Fig.1, under sufficient refrigeration, the z-axis direction wavelength of an electron dominates over the width of the semiconductor sample, because of the reduction in its kinetic energy. This implies that the angular momentum becomes a conserved quantity and thus a complete 2dimensional and circular motion will be formed. As a consequence, it is assumed that a de Broglie wave will be formed. In Fig. 1, assuming an electron forms a circle, the de Broglie condition is

$\mathrm{N} \lambda=2 \pi r_{0}$,

where $\mathrm{N}, \lambda$, and $\mathrm{r}_{0}$ denote an integer, the wavelength of an electron, and the radius of the circular motion in Fig. 1, respectively.

Thus, 
$\lambda=\frac{2 \pi r_{0}}{N}$.

Therefore, we see that the wave number, $\mathrm{k}$, is given by,

$\mathrm{k}=\frac{N}{2 \pi r_{0}}$.

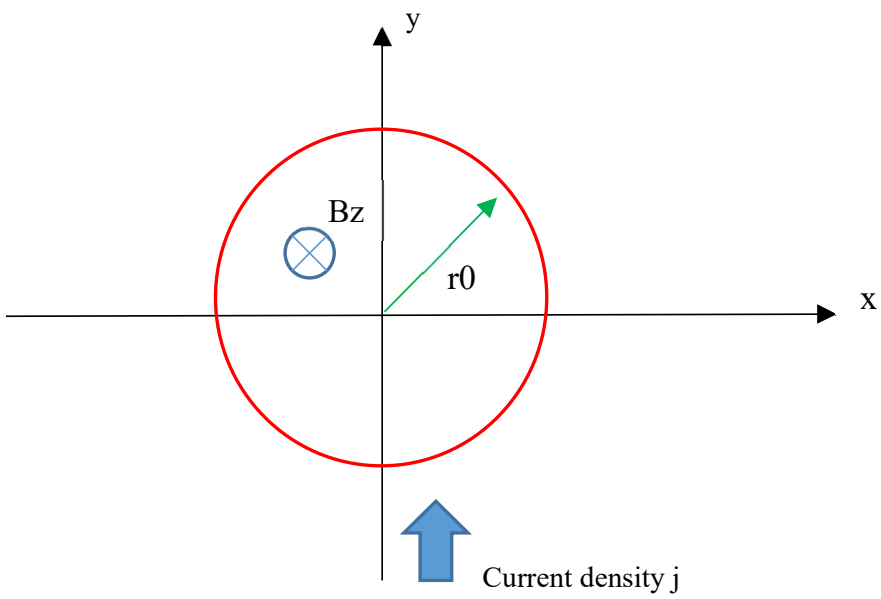

Fig. 1. Schematic of the model considered in this work. In this figure, a current flows along either $x$ - or $y$-axis, and a magnetic field is applied parallel with the z-axis. Due to the conservation of angular momentum, a rotation of electron and radius $\mathrm{r}_{0}$ are formed.

If the rotational wave function can be approximated as a plane wave, the velocity, $\mathrm{v}$, can be found to be,

$\mathrm{V}=\frac{\hbar k}{m}=\frac{\hbar}{m} \frac{N}{2 \pi r_{0}}$

where $\mathrm{m}$ denotes the mass of an electron. As mentioned, we assume that the electrical force, $\mathrm{F}_{\mathrm{E}}$, is balanced to the Lorentz force, $F_{B}$.

In addition to this balance, we consider the following cases:

(i) $\quad \overrightarrow{F_{B}}$ and $\vec{F}_{E}$ are parallel to each other.

(ii) $\quad \overrightarrow{F_{B}}$ and $\vec{F}_{E}$ are perpendicular to each other.

In case (i) a force balance can be formed. This implies that the resistivity, $\rho$, is anisotropic as claimed by the measurements. In this work, we consider only $\rho_{\mathrm{xy}}$. 
In case (i), from the force balance $\left|\overrightarrow{F_{B}}\right|=\left|\overrightarrow{F_{E}}\right|$,

$\mathrm{q} \frac{\hbar}{m} \frac{N}{2 \pi r_{0}} B_{z}=q E_{y}$,

where $B_{z}, q$, and $E_{y}$ denote the magnetic field, the charge of an electron, and the electric field in the system, respectively.

The following current, I, can be considered as,

$\mathrm{I}=\frac{Q}{T_{c}}=\frac{N^{\prime} q}{T_{c}}=\frac{\omega_{c}}{2 \pi} N^{\prime} q$

where $T_{c}, \omega_{c}$, and $Q$ denote the cyclotron period, the cyclotron angular frequency, and a general electric charge, respectively.

Importantly, in Eq. (6), the general electric charge, Q, is quantized by an integer, N' and electric charge of an electron, q.

Thus,

$\mathrm{I}=\frac{\omega_{c}}{2 \pi} N^{\prime} q=\frac{N^{\prime} q}{2 \pi} \frac{q B_{z}}{m}$.

Substituting $B_{z}$ into Eq. (7), we obtain,

$\mathrm{q} \frac{\hbar}{m} \frac{N}{2 \pi r_{0}} I \frac{2 \pi}{N^{\prime} q} \frac{m}{q}=q E_{y}$.

The voltage, $\mathrm{V}_{\mathrm{H}}$, is given by,

$\oint \vec{E} \cdot d \vec{s}=V_{H}$.

Thus,

$2 \pi r_{0} E_{y}=V_{H}$.

Therefore, we obtain,

$\hbar \frac{N}{N^{\prime}} I \times 2 \pi \frac{1}{q}=q E_{y} \times 2 \pi r_{0}=q V_{H}$.

The Hall resistance, $\mathrm{R}_{\mathrm{H}}$, is given by,

$R_{H}=\frac{V_{H}}{I}$.

Therefore, we find that,

$R_{H}=\frac{h}{q^{2}} \frac{N}{N^{\prime}}$.

In the fractional quantum Hall effect, the applied magnetic fields are stronger than that of the integer quantum Hall effect. This results in the integer $\mathrm{N}$ (which is the number of nodes along a stationary wave) can take a value greater than the number 1. By contrast, in the integer quantum Hall effect, because the applied magnetic fields are smaller than those in the fractional Hall effect, the integer $\mathrm{N}$ is always equal to 1 . The above equation for $\mathrm{R}_{\mathrm{H}}$ is valid for both the integer quantum Hall effect and the fractional quantum Hall effect. 


\section{Method}

As shown in Fig. 1, the system considered here exhibits a stationary wave with a radius $r_{0}$. The integer $\mathrm{N}$ is assumed to be the integer of the quantized magnetic flux, thus,

$\pi r_{0}^{2} B_{z}=N \frac{h}{Q}$

In this case, the derived expression for $\mathrm{R}_{\mathrm{H}}$, given in Eq. (13), forms a set of simultaneous equations and thus the charge $\mathrm{Q}$ should be quantized. We therefore see that,

$\pi r_{0}^{2} B_{z}=\frac{N}{N^{\prime}} \frac{h}{q}$

which yields

$B_{Z}=\frac{1}{\pi r_{0}^{2}} \frac{N}{N^{\prime}} \frac{h}{q}$

As discussed later in this work, the integer N' takes only odd values, while $\mathrm{N}$ can take any of the natural numbers. Note that $\mathrm{r}_{0}$ is assumed to be $2.5 \times 10^{-8} \mathrm{~m}$ in this paper.

Thus, for a given pair of ( $\left.N, N^{\prime}\right)$, the magnetic field, $B_{z}$, and Hall resistance, $R_{H}$, are obtained. In the next section of Result, we will see that this theory well predicts the experiment.

Among the pairs of $\mathrm{N}_{\text {and }} \mathrm{N}^{\prime}, \mathrm{N}=2$ and $\mathrm{N}^{\prime}=1$ is not permitted. In general, states are not permitted if following inequality is satisfied,

(node number) $\geq$ (electron number).

If the integer $\mathrm{N}$ were to be considered as the magnetic quantum number, the above inequality would imply that the number of states would be greater than the number of electrons, which would represent a contradiction. Thus, the pair of $\mathrm{N}=2$ and $\mathrm{N}^{\prime}=1$ is prohibited.

The reason that $\mathrm{N}^{\prime}$ in this paper takes only odd values is that in this work we do not consider Cooper pairs or superconductivity; if N' took even values it would imply the existence of Cooper pairs and superconductivity.

\section{Result}

In Fig. 2, the relationship between the plateaus in $\rho_{x y}$ for a given applied magnetic field is shown, which was obtained by the equations in Method and Theory section in the present paper. The agreement with the measurements given in Ref. $[10,12]$ is good. 


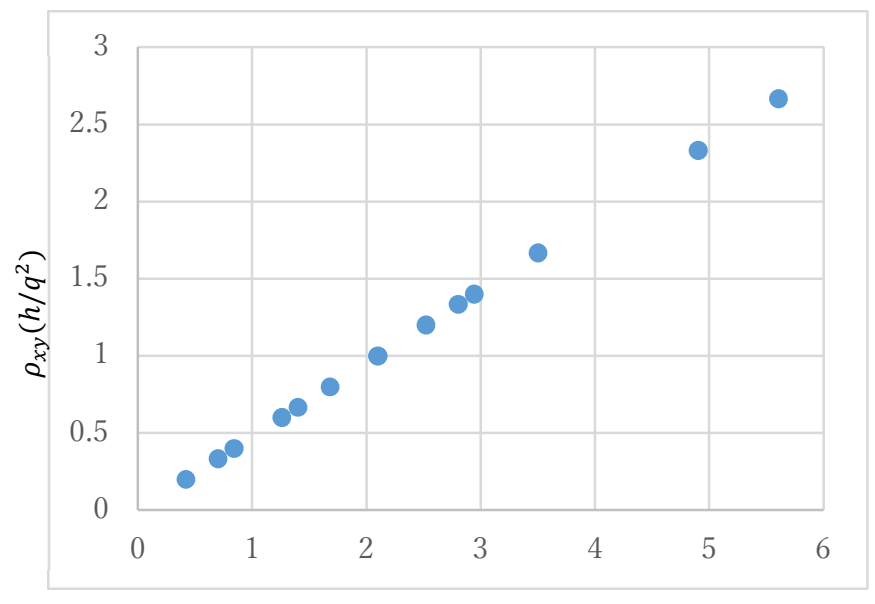

Applied magnetic field [T]

Fig. 2. The position of the plateau in the $\rho_{x y}$ plotted against the applied magnetic field. In this figure, each blue point for the magnetic fields indicates the position of plateau in the fractional quantum Hall effect. The integer $N^{\prime}$ is always odd; this figure shows the results obtained for $\mathrm{N}^{\prime}=3$ or 5 . The pair of values $\left(\mathrm{N}^{\prime}, \mathrm{N}^{\prime}\right)=(2,1)$ is prohibited. The agreement with measurements published in Ref. $[10,12]$ is good. It is noted that in the relatively large magnetic field, a slight error appears. This is because this figure shows the results only the values 3 and 5 .

\section{Discussion}

\subsection{The relation between the integer quantum Hall effect and the fractional quantum Hall effect}

This paper described both the integer quantum Hall effect and the fractional quantum Hall effect using a single model. It is widely held that the fractional quantum Hall effect requires many body interactions via Coulomb forces. Although Laughlin and Chern-Simons GL or fractional statistics describes the fractional quantum Hall effect to some degree, the ratio of $N$ to $N^{\prime}$ in the expression describing $\mathrm{R}_{\mathrm{H}}$, Eq. (13), cannot be determined by the coherent method. That is, they needed to use the hierarchy method, which is unnatural and complex. In particular, Laughlin theory can only be applied for systems where $\frac{N}{N^{\prime}}=1 / 3$; thus it is seen that currently there are no theories that obtain an expression for $\mathrm{R}_{\mathrm{H}}$ via a simple method. Although the continued fractional method, i.e., the hierarchy theory, has been suggested as a method to explain the fractional numbers, it must be noted that this method is a complex and unnatural theory. However, in this work a natural derivation of an expression for $\mathrm{R}_{\mathrm{H}}$ has been presented.

Many papers have suggested that the origin of the integer quantum Hall effect and the fractional 
quantum Hall effect are different. However, this work suggests that, while the fractional quantum Hall effect is general, the integer quantum Hall effect is simply a special case for the general fractional quantum Hall effect. Moreover, this paper claims the source of the integer and fractional quantum Hall effects is identical, i.e., the force balance of the Lorentz and electrical forces. The theory this work presents unifies the origin of the two effects. Previous theories have not been able to explain experiment in terms of the plateau in $\rho_{x y}$ for applied magnetic fields; whereas this work, however, has succeeded in describing these findings.

\subsection{Refrigeration and mobility}

Here we consider why both the integer quantum Hall effect and the fractional quantum Hall effect require refrigeration. If the refrigeration is insufficient, we cannot neglect motions parallel with the zaxis, i.e., the motions occur across three dimensions. This is because the wavelength of an electron does not dominate over the width of the sample owing to its high kinetic energy. Thus, to ensure that the wavelength dominates over the width of the sample, significant refrigeration is required. By doing this, the motion occurs only in two dimensions and the angular momentum is conserved.

We now consider why the fractional quantum Hall effect requires a relatively high mobility [11]. When the mobility is too small, impurities in the sample, which are causes of the low mobility, will increase. However, while the fractional quantum Hall effect requires a stationary wave to exist, the node of the stationary wave cannot exist at the same position as an impurity. Thus, if there are too many impurities in the sample, the stationary wave cannot be formed (except for in the case $\mathrm{N}=1$ ). This implies that the fractional quantum Hall effect cannot occur.

\subsection{Significance of this paper}

To date, the fractional quantum Hall effect and the integer quantum Hall effect have been independently explained. Only the fractional quantum Hall effect considers Coulomb body interactions between electrons. There exists, however, some experimental findings that are not well explained by theory. In our literature reviews, the plateau in $\rho_{x y}$ vs. applied magnetic field experiment was not explained theoretically. In this work, both the integer quantum Hall effect and the fractional quantum Hall effect can occur in the same system, thus this work suggests that both effects originate from the same source; this common cause of both effects is the force balance between the Lorentz force and the electrical force. Moreover, the two integers regarding the stationary wave and the quantization of charges have the concrete physical meanings. Thus, the significance of this work is that a clear physical picture of the origin of both effects is obtained. This theory also explains experimentally observed features that have to date not been explained. 


\section{Conclusion}

In this paper, both the integer quantum Hall effect and the fractional quantum Hall effect were described simultaneously; both effects were explained as originating from a single mechanism. In this process, the fractional numbers of the fractional quantum Hall effect were naturally derived and the integer quantum Hall effect was found to be a special case of the fractional quantum Hall effect. This paper also succeeded in describing quantitatively the plateaus in $\rho_{x y}$ vs. applied magnetic field measurements.

\section{References}

[1] K. von Klitzing, G. Dorda and M. Pepper. Phys. Rev. Lett. 45, 494 (1980)

[2] D.C Tsui, H.L Stormer and A.C. Gossard, Phys. Rev. Lett. 48, 1559 (1982)

[3] R.B. Laughlin, Phys. Rev. Lett. B23, 5632 (1981)

[4] R. B. Laughlin, Phys. Rev. Lett. 50, 1395 (1983)

[5] S.C. Zhang, In. J. of Mod. Phys. B6, 25 (1992)

[6] S. Das Sarma, A. Pinczuk, Perspective in Quantum Hall Effect (John Wiley and Sons) (1997)

[7] M. Stone, Quantum Hall Effect (World Scientific) (1992)

[8] N. Read, Phys. Rev. Lett. 62, 86 (1989)

[9] S.M. Girvin and A. H. MacDonald, Phys. Rev. Lett. 58, 1252 (1987)

[10] R. Willett, et al, Phys. Rev. Lett. 59, 1776 (1987)

[11] D. Yoshioka, "Quantum Hall effect”, (Iwanami Shoten, Tokyo) (2016)

[12] C. Kittel, "Introduction to solid state physics", Japanese version 8, p. 534, Maruzen in Tokyo (2005)

\section{Additional information}

This paper is not related to any competing interests such as funding, employment, and personal financial interests. Moreover, this paper is not related to non-financial competing interests.

\section{Acknowledgement}

We thank Enago (www.enago.jp) for the English language review. 